DOI: https://doi.org/10.24297/jam.v20i.9056

\title{
Metallic Means : Beyond the Golden Ratio, New Mathematics and Geometry of all Metallic Ratios based upon Right Triangles, The Formation of the "Triads" of Metallic Means, And their Classical Correspondence with Pythagorean Triples and $p \equiv 1(\bmod 4)$ Primes, Also the Correlation between Metallic Numbers and the Digits 369 , Triangles - Triads - Triples - \& 369

\author{
Dr. Chetansing Rajput
}

M.B.B.S. Nair Hospital (Mumbai University) India, Asst. Commissioner (Govt. of Maharashtra)

Lecture Link: https://youtu.be/vBfVDaFnA2k

Email: chetansingkrajput@gmail.com

Website: https://goldenratiorajput.com/

\begin{abstract}
This paper brings together the newly discovered generalised geometry of all Metallic Means and the recently published mathematical formulae those provide the precise correlations between different Metallic Ratios. The paper also puts forward the concept of the "Triads of Metallic Means". This work also introduces the close correspondence between Metallic Ratios and the Pythagorean Triples as well as Pythagorean Primes. Moreover, this work illustrates the intriguing relationship between Metallic Numbers and the Digits 369.
\end{abstract}

Keywords: Fibonacci sequence, Pi, Phi, Pythagoras Theorem, Divine Proportion, Silver Ratio, Golden Mean, Right Triangle, Metallic Numbers, Pell Numbers, Lucas Numbers, Golden Proportion, Metallic Ratio, Metallic Triples, 369 , Pythagorean Triples, Pythagorean Primes, Golden Ratio, Metallic Mean

\section{Introduction}

The prime objective of this work is to synergize the following couple of newly discovered aspects of Metallic Means:

1) The Generalised Geometric Construction of all Metallic Ratios: cited by Wikipedia in its page on "Metallic Mean"[1]. This generalised geometric substantiation of all Metallic Means was published in January 2021 [5]

2) The Mathematical Formula that provides the precise correlation between different Metallic Means. This explicit formula has been recently published in the month of May 2021 [6]

The synergism between above two features of Metallic Means unveils an intriguing pattern of Metallic Ratios, which asserts that the mathematical implications of these Means have not been fully appreciated so far. 
Is Golden Ratio to be perceived only in handful of Botanical formations ?..... and the Silver Ratio just to be observed in the man-made $A 4, A 5$, A6 paper sizes.....?

Nope! The whole family of Metallic Ratios is certainly far more important than that. And, the abovementioned Geometry and Mathematics synergically enables us to recognize the full worth of these Metallic Means, as follows.

As a brief introduction, each Metallic Mean $\boldsymbol{\delta}_{\mathbf{n}}$ is the root of the simple Quadratic Equation $\mathbf{X}^{\mathbf{2}}-\mathbf{n} \mathbf{X}-\mathbf{1}=\mathbf{0}$, where $\mathbf{n}$ is any positive natural number.

Thus, the fractional expression of the $\mathrm{n}^{\text {th }}$ Metallic Ratio is $\boldsymbol{\delta}_{n}=\frac{\mathbf{n}+\sqrt{\mathbf{n}^{2}+\mathbf{4}}}{\mathbf{2}}$

Moreover, each Metallic Ratio can be expressed as the continued fraction:

$$
\boldsymbol{\delta}_{\mathbf{n}}=\mathbf{n}+\frac{\mathbf{1}}{\mathbf{n}+\frac{\mathbf{1}}{\mathbf{n}+\frac{\mathbf{1}}{\mathbf{n}+\ldots}}} ; \text { And hence, } \boldsymbol{\delta}_{\mathbf{n}}=\mathbf{n}+\frac{\mathbf{1}}{\boldsymbol{\delta} \mathbf{n}}
$$

\section{GEOMETRIC CONSTRUCTION OF ALL METALLIC MEANS :}

Golden Ratio in regular Pentagon, and Silver Ratio in regular Octagon, but contrary to natural expectations, in Polygons, Diagonals, and the Bronze Mean, Antonia Redondo Buitrago clearly demonstrated that the Bronze Mean does not appear as a side to diagonal relationship in a polygon [7] [8]. And for long time, there remained the question of whether or not the higher Metallic Means appear as a ratio between line segments? Now the question has been answered positively. [1] [5]

Each Metallic Ratio can be constructed geometrically with a special Right Angled Triangle. Any $\mathrm{n}^{\text {th }}$ Metallic Mean can be represented by the Right Triangle having its catheti $\mathbf{1}$ and $\frac{\mathbf{n}}{\mathbf{2}}$. Hence, the right triangle with one of its catheti $=\mathbf{1}$ may substantiate any Metallic Mean, having its second cathetus $=\frac{\mathbf{n}}{\mathbf{2}}$, where $n=1$ for Golden Ratio, $n=2$ for Silver Ratio, $n=3$ for Bronze Ratio, and so on. Such Right Triangle provides the precise value of $n^{\text {th }}$ Metallic Mean by the generalised formula:

$$
\text { The } n^{\text {th }} \text { Metallic Mean }\left(\boldsymbol{\delta}_{n}\right)=\text { Hypotenuse }+ \text { Cathetus } \frac{n}{2}
$$

Such Right Triangle not only provides for the accurate geometric construction and precise fractional expression of any $\mathrm{n}^{\text {th }}$ Metallic Mean $\left(\boldsymbol{\delta}_{\mathbf{n}}\right)$, but its every geometric feature is the prototypical form of that Metallic Mean [5] [9] [10]. The characteristic geometry of such Right Triangle having its catheti $\mathbf{1}$ and $\frac{\mathbf{n}}{\mathbf{2}}$, is resplendent with the corresponding $\mathrm{n}^{\text {th }}$ Metallic Mean $\left(\boldsymbol{\delta}_{\mathbf{n}}\right)$ embedded in its every aspect. For example, the remarkable expression of Golden Ratio in every geometric feature of 1:2: $\sqrt{5}$ triangle, including all its angles and side lengths, its 'Incenter-Excenters Orthocentric system', its Gergonne and Nagel triangles, and also the Nobbs points and the Gergonne line, various triangle centers as well as the Incircle of $1: 2: \sqrt{5}$ triangle, make this triangle the quintessential form of the Golden Ratio $(\varphi)$ and also of the fourth Metallic Mean $\left(\varphi^{3}\right)$. [5] 


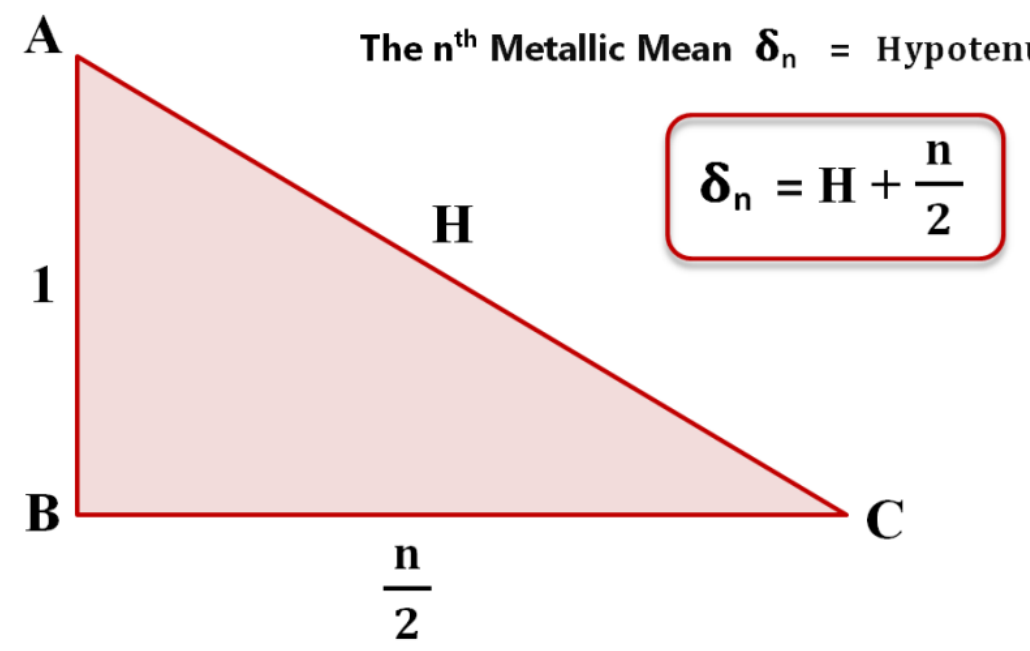

Figure 1: Generalised Right Triangle to represent any $\mathrm{n}^{\text {th }}$ Metallic Mean $\boldsymbol{\delta}_{\mathrm{n}}$

\section{MATHEMATICAL CORRELATIONS AMONG DIFFERENT METALLIC RATIOS :}

If $\mathbf{K}, \mathbf{m}$ and $\mathbf{n}$ are three positive integers such that $\frac{\mathbf{m n}+\mathbf{4}}{\mathbf{m}-\mathbf{n}}=\mathbf{k}$

then, it is observed that

$\frac{\boldsymbol{\delta}_{\mathrm{m}} \times \boldsymbol{\delta}_{\mathrm{n}}+\mathbf{1}}{\boldsymbol{\delta}_{\mathrm{m}}-\boldsymbol{\delta}_{\mathrm{n}}}=\boldsymbol{\delta}_{\mathrm{k}}$ where $\boldsymbol{\delta}_{\mathrm{k}}, \boldsymbol{\delta}_{\mathrm{m}}$ and $\boldsymbol{\delta}_{\mathrm{n}}$ are the $\mathrm{k}^{\text {th }}, \mathrm{m}^{\text {th }}$ and $\mathrm{n}^{\text {th }}$ Metallic Means respectively. This explicit formula, among the several other formulae those give the precise mathematical relations between different Metallic Means, has been recently published in the work mentioned in Reference: [6]

\section{METALLIC MEANS' “TRIADS" :}

The abovementioned explicit formula gives the "Triads" of Metallic Means, as $\left[\boldsymbol{\delta}_{\mathrm{n}}, \boldsymbol{\delta}_{\mathrm{m}}, \boldsymbol{\delta}_{\mathrm{k}}\right.$,

where $\frac{\mathbf{m n}+\mathbf{4}}{\mathbf{m}-\mathbf{n}}=\mathbf{k}$ and $\frac{\mathbf{k n}+\mathbf{4}}{\mathbf{k}-\mathbf{n}}=\mathbf{m}$

hence, $\frac{\boldsymbol{\delta}_{\mathrm{m}} \times \boldsymbol{\delta}_{\mathbf{n}}+\mathbf{1}}{\boldsymbol{\delta}_{\mathrm{m}}-\boldsymbol{\delta}_{\mathbf{n}}}=\boldsymbol{\delta}_{\mathrm{k}}$ and also $\frac{\boldsymbol{\delta}_{\mathbf{k}} \times \boldsymbol{\delta}_{\mathbf{n}}+\mathbf{1}}{\boldsymbol{\delta}_{\mathbf{k}}-\boldsymbol{\delta}_{\mathbf{n}}}=\boldsymbol{\delta}_{\mathrm{m}}$

For example, if $n=6$, the three integers 6,11 and 14 satisfy the prerequisite $\frac{\mathbf{m n}+\mathbf{4}}{\mathbf{m}-\mathbf{n}}=\mathbf{k}$; Hence, the three Merallic means $\boldsymbol{\delta}_{6}, \boldsymbol{\delta}_{11}$ and $\boldsymbol{\delta}_{14}$ form a Triad, such that $\frac{\boldsymbol{\delta}_{11} \times \boldsymbol{\delta}_{6}+\mathbf{1}}{\boldsymbol{\delta}_{11}-\boldsymbol{\delta}_{6}}=\boldsymbol{\delta}_{\mathbf{1 4}}$ and also $\frac{\boldsymbol{\delta}_{14} \times \boldsymbol{\delta}_{6}+\mathbf{1}}{\boldsymbol{\delta}_{14}-\boldsymbol{\delta}_{6}}=\boldsymbol{\delta}_{\mathbf{1 1}}$ 
Just like $n=6$ exemplified above, every integer forms such multiple triads, as shown below for first ten integers.

Table 1: "Triads" of Metallic Means formed by the First Ten Metallic Means:

\begin{tabular}{|c|c|c|c|c|c|c|c|c|}
\hline $\mathbf{n}$ & 1 & 1 & 1 & 1 & 1 & 1 & 1 & 1 \\
\hline $\mathrm{m}$ & $\mathbf{2}$ & $\mathbf{6}$ & & & & & & \\
\hline $\mathrm{k}$ & $\mathbf{6}$ & $\mathbf{2}$ & & & & & & \\
\hline
\end{tabular}

\begin{tabular}{|l|l|l|l|l|l|l|l|l|}
\hline$n$ & 2 & 2 & 2 & 2 & 2 & 2 & 2 & 2 \\
\hline$m$ & 3 & 4 & 6 & 10 & & & & \\
\hline$k$ & 10 & 6 & 4 & 3 & & & & \\
\hline
\end{tabular}

\begin{tabular}{|l|l|l|l|l|l|l|l|l|}
\hline$n$ & 3 & 3 & 3 & 3 & 3 & 3 & 3 & 3 \\
\hline$m$ & 4 & 16 & & & & & & \\
\hline$k$ & 16 & 4 & & & & & & \\
\hline
\end{tabular}

\begin{tabular}{|l|l|l|l|l|l|l|l|l|}
\hline $\mathrm{n}$ & 4 & 4 & 4 & 4 & 4 & 4 & 4 & 4 \\
\hline $\mathrm{m}$ & 5 & 6 & 8 & 9 & 14 & 24 & & \\
\hline $\mathrm{k}$ & 24 & 14 & 9 & 8 & 6 & 5 & & \\
\hline
\end{tabular}

\begin{tabular}{|l|l|l|l|l|l|l|l|l|}
\hline $\mathrm{n}$ & 5 & 5 & 5 & 5 & 5 & 5 & 5 & 5 \\
\hline $\mathrm{m}$ & 6 & 34 & & & & & & \\
\hline $\mathrm{k}$ & 34 & 6 & & & & & & \\
\hline
\end{tabular}

\begin{tabular}{|c|l|l|l|l|l|l|l|l|}
\hline$n$ & 6 & 6 & 6 & 6 & 6 & 6 & 6 & 6 \\
\hline $\mathrm{m}$ & 7 & 8 & 10 & 11 & 14 & 16 & 26 & 46 \\
\hline $\mathrm{k}$ & 46 & 26 & 16 & 14 & 11 & 10 & 8 & 7 \\
\hline
\end{tabular}

: Shaded Triads have been exemplified above. 


\begin{tabular}{|l|l|l|l|l|l|l|l|l|}
\hline $\mathrm{n}$ & 7 & 7 & 7 & 7 & 7 & 7 & 7 & 7 \\
\hline $\mathrm{m}$ & 8 & 60 & & & & & & \\
\hline $\mathrm{k}$ & 60 & 8 & & & & & & \\
\hline
\end{tabular}

\begin{tabular}{|l|l|l|l|l|l|l|l|l|}
\hline $\mathrm{n}$ & 8 & 8 & 8 & 8 & 8 & 8 & 8 & 8 \\
\hline $\mathrm{m}$ & 9 & 10 & 12 & 25 & 42 & 76 & & \\
\hline $\mathrm{k}$ & 76 & 42 & 25 & 12 & 10 & 9 & & \\
\hline
\end{tabular}

\begin{tabular}{|l|l|l|l|l|l|l|l|l|}
\hline n & 9 & 9 & 9 & 9 & 9 & 9 & 9 & 9 \\
\hline m & 10 & 14 & 26 & 94 & & & & \\
\hline k & 94 & 26 & 14 & 10 & & & & \\
\hline
\end{tabular}

\begin{tabular}{|l|l|l|l|l|l|l|l|l|}
\hline $\mathrm{n}$ & 10 & 10 & 10 & 10 & 10 & 10 & 10 & 10 \\
\hline $\mathrm{m}$ & 11 & 12 & 14 & 18 & 23 & 36 & 62 & 114 \\
\hline $\mathrm{k}$ & 114 & 62 & 36 & 23 & 18 & 14 & 12 & 11 \\
\hline
\end{tabular}

It may be noticed from above Table that every $\mathrm{n}^{\text {th }}$ Metallic Mean can give precise values of various Metallic Means by the formula $\frac{\boldsymbol{\delta}_{\mathbf{m}} \times \boldsymbol{\delta}_{\mathbf{n}}+\mathbf{1}}{\boldsymbol{\delta}_{\mathbf{m}}-\boldsymbol{\delta}_{\mathbf{n}}}=\boldsymbol{\delta}_{\mathrm{k}}$, maximum upto $\left(\mathrm{n}^{2}+\mathrm{n}+4\right)^{\text {th }}$ Metallic Mean:

$m_{\max }=k_{\max }=\left(n^{2}+n+4\right)$

Also noticeably, the Even Integers $\left(\right.$ Even $\mathrm{n}_{\mathrm{s}}$ ) form comparatively more Triads than the Odd $\mathrm{n}_{\mathrm{s}}$. Several such patterns about these Triads will be discussed in detail, in the subsequent parts of this paper.

More importantly, such Triads can be represented geometrically, as shown below. 
For instance, the Triad [ $\boldsymbol{\delta}_{\mathbf{n}}, \boldsymbol{\delta}_{\mathbf{m}}, \boldsymbol{\delta}_{\mathbf{k}}$ ] is illustrated geometrically in following Figure $\mathbf{2}$.

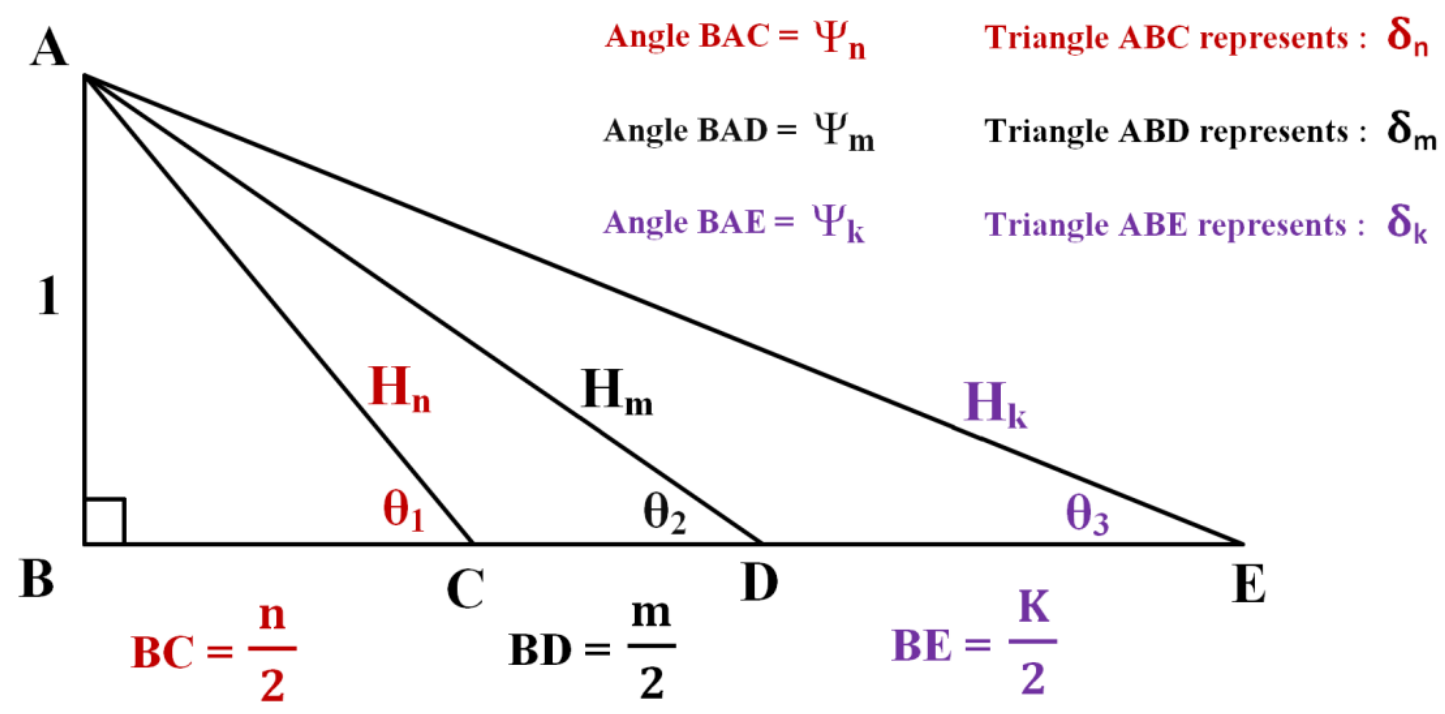

Figure 2: Three Right Triangles representing the "Triad of Metallic Means"

Remarkably, in above Figure 2: if the three Metallic Means $\boldsymbol{\delta}_{\mathbf{n}} \boldsymbol{\delta}_{\mathbf{m}}$ and $\boldsymbol{\delta}_{\mathbf{k}}$ constitute a Triad, then

$\boldsymbol{\theta}_{1}=\boldsymbol{\theta}_{2}+\boldsymbol{\theta}_{3} \quad\left(\right.$ and also $\left.\boldsymbol{\Psi}_{\mathrm{n}}+90^{0}=\boldsymbol{\Psi}_{\mathrm{m}}+\boldsymbol{\Psi}_{\mathrm{k}}\right)$

In other words,

$\arctan \frac{2}{n}=\arctan \frac{2}{m}+\arctan \frac{2}{k}$

Solving it gives the couple of correlations: $\frac{\mathbf{m n}+\mathbf{4}}{\mathbf{m}-\mathbf{n}}=\mathbf{k}$ and $\frac{\mathbf{k n}+\mathbf{4}}{\mathbf{k}-\mathbf{n}}=\mathbf{m}$

And hence satisfy the prerequisite for $\frac{\boldsymbol{\delta}_{\mathrm{m}} \times \boldsymbol{\delta}_{\mathbf{n}}+\mathbf{1}}{\boldsymbol{\delta}_{\mathrm{m}}-\boldsymbol{\delta}_{\mathbf{n}}}=\boldsymbol{\delta}_{\mathrm{k}}$ and also $\frac{\boldsymbol{\delta}_{\mathrm{k}} \times \boldsymbol{\delta}_{\mathbf{n}}+\mathbf{1}}{\boldsymbol{\delta}_{\mathrm{k}}-\boldsymbol{\delta}_{\mathbf{n}}}=\boldsymbol{\delta}_{\mathrm{m}}$

Moreover, entire geometry of such Triad-Triangles is resplendent with the precise correlation among the three Metallic Means $\boldsymbol{\delta}_{\mathbf{n}}, \boldsymbol{\delta}_{\mathbf{m}}$ and $\boldsymbol{\delta}_{\mathbf{k}}$. Following intriguing relations are observed in above Figure 2. Consider the larger acute angles of the three triangles;

$$
\begin{aligned}
& \Psi_{\mathrm{m}}+\Psi_{\mathrm{k}}=2 \arctan \boldsymbol{\delta}_{\mathrm{n}} \\
& \Psi_{\mathrm{m}}-\Psi_{\mathrm{n}}=2 \arctan \frac{1}{\delta_{\mathrm{k}}} \\
& \Psi_{\mathrm{k}}-\Psi_{\mathrm{n}}=2 \arctan \frac{1}{\delta_{\mathrm{m}}}
\end{aligned}
$$


Also, it is observed that, $\Psi_{\mathrm{k}}-\Psi_{\mathrm{m}}=2 \arctan \frac{1}{\delta_{\mathrm{x}}}$ where $\mathbf{x}=\frac{\mathbf{k m}+\mathbf{4}}{\mathbf{k}-\mathbf{m}}$ and the mean $\boldsymbol{\delta}_{\mathrm{x}}$ lies somewhere between $\boldsymbol{\delta}_{\mathrm{k}}$ and $\boldsymbol{\delta}_{\mathrm{m}}$. However, unlike $\mathrm{n}, \mathrm{m}$ and $\mathrm{k}$, this $\mathrm{X}$ may or may not be an integer. In certain cases, the $\mathrm{X}$ is an integer, for example, in case of $n=4$ and the Triad $\left[\boldsymbol{\delta}_{4}, \boldsymbol{\delta}_{6}, \boldsymbol{\delta}_{14}\right.$ ], the value of $X$ is 11 , and hence interestingly enough, it forms new Triad of Metallic Means $\left[\boldsymbol{\delta}_{6}, \boldsymbol{\delta}_{11}, \boldsymbol{\delta}_{14}\right]$ with $\mathrm{n}=6$ i.e. a new $[\mathrm{m}, \mathrm{x}, \mathrm{k}]$ Triad is generated from [n, $\mathrm{m}, \mathrm{k}$ ].

Moreover, the Hypotenuses of the Triad-Triangles: $H_{n}, H_{m}$ and $H_{k}$ in Figure 2 exhibit following relations.

$\frac{\mathbf{H}_{\mathrm{k}}}{\mathbf{H}_{\mathrm{m}}}=\frac{2}{\mathbf{m}-\mathbf{n}} \times \mathbf{H}_{\mathrm{n}}$

However, more interesting aspect of this geometry of Metallic Means is that they exhibit a classical correspondence with Primitive Pythagorean Triples.

\section{Right Triangle for $\mathbf{n}^{\text {th }}$ Metallic Mean and the Primitive Pythagorean Triples :}

Each of the Right Triangles representing various Metallic Means, and hence the abovementioned Triads of Metallic Means are associated with a particular primitive Pythagorean triple, depending upon the value of $\mathbf{n}$. For example, Right Triangle for $6^{\text {th }}$ Metallic Mean $\left(\boldsymbol{\delta}_{6}\right)$, and hence, all Triads with $n=6$ are associated with 3-4-5 Pythagorean Triple. For instance, consider the Triad $\left[\boldsymbol{\delta}_{6}, \boldsymbol{\delta}_{11}, \boldsymbol{\delta}_{14}\right]$. If this Triad is constructed geometrically, as in above Figure 2, then it is observed that:

$\mathbf{2 \theta}_{\mathbf{1}}=\left(\boldsymbol{\theta}_{1}+\boldsymbol{\theta}_{2}+\boldsymbol{\theta}_{3}\right)=$ The Smaller Acute Angle of 3-4-5 Pythagorean Triangle i.e. $\arctan \frac{\mathbf{3}}{\mathbf{4}}$

$2 \Psi_{n}-\mathbf{9 0}^{0}=\left(\Psi_{n}+\Psi_{m}+\Psi_{k}\right)-180^{\circ}=$ The Larger Acute Angle of 3-4-5 Pythagorean Triangle i.e. $\arctan \frac{\mathbf{4}}{3}$

Likewise, Right Triangle for $3^{\text {rd }}$ Metallic Mean (and all Triads with $n=3$ ), or Right Triangle for $10^{\text {th }}$ Metallic Mean (and Triads with $\mathrm{n}=10$ ), are all related to 5-12-13 Pythagorean Triple, and so on.

Consider the fractional expression of the $n^{\text {th }}$ Metallic Mean : $\boldsymbol{\delta}_{n}=\frac{n+\sqrt{n^{2}+4}}{2}$

The radical $\left(\mathbf{n}^{2}+4\right)$ in this Fractional expression of $\boldsymbol{\delta}_{n}$ determines the Pythagorean Triple associated with the Right Triangle representing a Metallic Mean $\left(\boldsymbol{\delta}_{\mathbf{n}}\right)$. Any such Right Triangle representing a Metallic Mean $\boldsymbol{\delta}_{\mathbf{n}}$ is associated with such Primitive Pythagorean Triple whose Hypotenuse is Factor of the Radical $\left(\mathbf{n}^{\mathbf{2}}+\mathbf{4}\right)$, with following observed subrules :

If $\mathbf{n}$ is Odd : Hypotenuse of associated Pythagorean Triple $=\left(\mathbf{n}^{\mathbf{2}}+\mathbf{4}\right)$

If $\mathbf{n}$ is Even and multiple of Four, $\mathbf{n}=\mathbf{4 x}$ : Hypotenuse of associated Pythagorean Triple $=\mathbf{n x}+\mathbf{1}$

If $\mathbf{n}$ is Even but not Multiple of Four : Smaller Cathetus of associated Pythagorean Triple $=\frac{\mathbf{n}}{\mathbf{2}}$

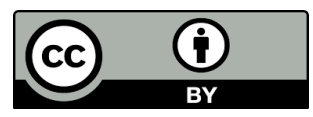


Following Table 2 shows associated Pythagorean Triples for first few values of $n$ :

Table 2: Primitive Pythagorean Triples associated with the Right Triangles for Metallic Means: depenending upon $\mathbf{n}$

\begin{tabular}{|c|c|}
\hline $\mathbf{n}$ & Associated Pythagorean Triple \\
\hline 1 & $3-4-5$ \\
\hline 2 & None \\
\hline 3 & $5-12-13$ \\
\hline 4 & $3-4-5$ \\
\hline 5 & $20-21-29$ \\
\hline 6 & $3-4-5$ \\
\hline 7 & $28-45-53$ \\
\hline 8 & $8-15-17$ \\
\hline 9 & $36-77-85$ \\
\hline 10 & $5-12-13$ \\
\hline 11 & $44-117-125$ \\
\hline 12 & $12-35-37$ \\
\hline 13 & $52-165-173$ \\
\hline 14 & $7-24-25$ \\
\hline
\end{tabular}

The classical correspondence between these Right Triangles for Metallic Means and the corresponding Primitive Pythagorean Triple is manifested as follows.

Consider again the Figure 2 representing the Triad of Metallic Means $\left[\boldsymbol{\delta}_{\mathbf{n}}, \boldsymbol{\delta}_{\mathbf{m}}, \boldsymbol{\delta}_{\mathbf{k}}\right]$.

$\mathbf{2 \theta _ { 1 }}=\left(\boldsymbol{\theta}_{1}+\boldsymbol{\theta}_{2}+\boldsymbol{\theta}_{3}\right)=$ The Smaller Acute Angle of associated Pythagorean Triple.

Similarly, $2 \Psi_{\mathbf{n}}-\mathbf{9 0} \mathbf{0}^{\mathbf{0}}=\left(\boldsymbol{\Psi}_{\mathbf{n}}+\boldsymbol{\Psi}_{\mathbf{m}}+\boldsymbol{\Psi}_{\mathbf{k}}\right)-\mathbf{1 8 0 ^ { \circ }}=$ The Larger Acute Angle of associated Pythagorean Triple.

\section{Special cases :}

1) For $\mathbf{n = 1}$ : the right Triangle that represents Golden Ratio has its Cathetus 1 longer than its Second Cathetus $\frac{\mathrm{n}}{2}$ Hence, for the Right Triangle representing Golden Ratio, and the Triad formed with $\mathrm{n}=1\left[\boldsymbol{\delta}_{1}, \boldsymbol{\delta}_{2}, \boldsymbol{\delta}_{6}\right]$ :

$\mathbf{2 \theta}_{\mathbf{1}}-\mathbf{9 0 ^ { \circ }}=\left(\theta_{1}+\theta_{2}+\theta_{3}\right)-90^{\circ}=$ The Smaller Acute Angle of associated Pythagorean Triple 3-4-5. And, $\mathbf{2} \Psi_{n}=\left(\Psi_{n}+\Psi_{m}+\Psi_{k}\right)-90^{\circ}=$ The Larger Acute Angle of associated Pythagorean Triple.

2) Similarly, in case of $\mathbf{n = 2}$ : the Triangle representing Silver Ratio is an Isosceles Right Triangle. Hence, for Triad formed with $\mathrm{n}=2$, like $\left[\boldsymbol{\delta}_{2}, \boldsymbol{\delta}_{3}, \boldsymbol{\delta}_{10}\right]$ or $\left[\boldsymbol{\delta}_{2}, \boldsymbol{\delta}_{4}, \boldsymbol{\delta}_{6}\right]$ :

$2 \boldsymbol{\theta}_{\mathbf{1}}=\left(\theta_{1}+\theta_{2}+\theta_{3}\right)=\mathbf{9 0}^{\circ}$,

and $2 \Psi_{\mathrm{n}}+90^{\circ}=\left(\Psi_{\mathrm{n}}+\Psi_{\mathrm{m}}+\Psi_{\mathrm{k}}\right)=180^{\circ}$

And hence there is no associated Pythagorean Triple for $n=2$. 


\section{3) For $\mathbf{n}=\mathbf{3}$ or $\mathbf{4}$ :}

$\mathbf{2 \theta}_{\mathbf{1}}=\left(\theta_{1}+\theta_{2}+\theta_{3}\right)=$ The Larger Acute Angle of associated Pythagorean Triple.

And, $2 \Psi_{n}-\mathbf{9 0}^{0}=\left(\Psi_{n}+\Psi_{m}+\Psi_{k}\right)-180^{\circ}=$ The Smaller Acute Angle of associated Pythagorean Triple.

Note the Right Triangles those represent the First and Fourth Metallic Means: $\boldsymbol{\delta}_{\mathbf{1}}$ ( that is Golden Ratio $\boldsymbol{\varphi}$ ) and $\boldsymbol{\delta}_{\mathbf{4}}$ (which equals $\varphi^{\mathbf{3}}$ ), are similar triangles.

In all the Right Triangles those represent $5^{\text {th }}$ Mean onwards, and Triads formed with $\mathrm{n}=5$ onwards, $(\mathrm{n}=5,6,7 \ldots .$. and so on) : $\mathbf{2} \boldsymbol{\theta}_{\mathbf{1}}=\left(\boldsymbol{\theta}_{\mathbf{1}}+\boldsymbol{\theta}_{\mathbf{2}}+\boldsymbol{\theta}_{\mathbf{3}}\right)=$ The Smaller Acute Angle of associated Primitive Pythagorean Triple ;

$2 \Psi_{n}-\mathbf{9 0}^{0}=\left(\Psi_{n}+\Psi_{m}+\Psi_{k}\right)-180^{\circ}=$ The Larger Acute Angle of associated Primitive Pythagorean Triple

In other words, on doubling the smaller acute angle of the $1: \frac{\mathrm{n}}{2}$ Right Triangle for any nth Metallic Mean, the Hypotenuse gets squared, to produce the associated Pythagorean Triple. For example, if the $26.565^{\circ}$ angle of $1: \frac{1}{2}$ triangle for Golden Ratio is doubled to $53.13^{\circ}$ : the associated 3-4-5 Pythagorean Triple is produced.

Moreover, an intriguing relation is also observed between the Hypotenuses of the Three Triangles in Figure 2 ( viz. $\mathrm{H}_{\mathrm{n}}, \mathrm{H}_{\mathrm{m}}$ and $\mathrm{H}_{\mathrm{k}}$ ) and the Hypotenuse of associated Pythagorean Triple $\mathbf{H}$.

If $\mathrm{n}$ is Odd: $\frac{\mathrm{H}_{\mathrm{k}}}{\mathrm{H}_{\mathrm{m}}} \times(\mathrm{m}-\mathrm{n})=\sqrt{\mathrm{H}}$

If $\mathrm{n}$ is Even and multiple of Four: $\frac{\mathrm{H}_{\mathrm{k}}}{\mathrm{H}_{\mathrm{m}}} \times(\mathrm{m}-\mathrm{n})=2 \sqrt{\mathrm{H}}$

If $\mathrm{n}$ is Even but not Multiple of Four: $\frac{\mathrm{H}_{\mathrm{k}}}{\mathrm{H}_{\mathrm{m}}} \times(\mathrm{m}-\mathrm{n})=2 \sqrt{2} \sqrt{\mathrm{H}}$

Thus, consider the following couple of facts. First: the generalised geometric substantiation of all Metallic Means based upon Right Triangles, as elaborated in the work mentioned in References [1] and [5]. And second: the close correspondence between the Right Triangles representing Metallic Ratios and the Primitive Pythagorean Triples, as describeded here. These couple of facts clearly highlight the underlying proposition that the Metallic Means are more closely associated with; and more holistically represented by the "Right Angled Triangles", rather than Pentagon, Octagon or any other $\left(n^{2}+4\right)$-gon.

\section{Metallic Means and Pythagorean Primes : The Prime Families of Metallic Means}

From the close correspondence between Metallic Means and Pythagorean Triples described so far, it becomes obvious that various Metallic Means are also closely associated with different Pythagorean Primes.

Consider the radical $\left(\mathbf{n}^{2}+\mathbf{4}\right)$ in the Fractional expression of the $\mathrm{n}^{\text {th }}$ Metallic Mean $\left(\boldsymbol{\delta}_{\mathrm{n}}\right)$. By Fermat's Theorem on Sums of Two Squares, this radical is an integer multiple of a prime of the form $p \equiv 1(\bmod 4)$. The Greatest Prime Factor of this radical $\left(n^{2}+4\right)$ is a Pythagorean Prime, as shown below in Table 3. 


\begin{tabular}{|c|c|c|}
\hline $\mathbf{n}$ & $n^{2}+4$ & $\begin{array}{l}\text { Greatest Prime Factor } \\
\qquad \text { of }\left(n^{2}+4\right) \text { : } \\
\text { A Pythagorean Prime }\end{array}$ \\
\hline 1 & 5 & 5 \\
\hline 2 & 8 & 2 \\
\hline 3 & 13 & 13 \\
\hline 4 & 20 & 5 \\
\hline 5 & 29 & 29 \\
\hline 6 & 40 & 5 \\
\hline 7 & 53 & 53 \\
\hline 8 & 68 & 17 \\
\hline 9 & 85 & 17 \\
\hline 10 & 104 & 13 \\
\hline 11 & 125 & 5 \\
\hline 12 & 148 & 37 \\
\hline 13 & 173 & 173 \\
\hline 14 & 200 & 5 \\
\hline 15 & 229 & 229 \\
\hline 16 & 260 & 13 \\
\hline
\end{tabular}

Table 3: The Greatest Prime Factors of the radical $\left(n^{2}+4\right)$

It is noticeable from above table that various values of $n$ have common Greatest Prime Factor of $\left(n^{2}+4\right)$.

For example, for $3^{\text {th }}, 10^{\text {th }}$ and $16^{\text {th }}$ Metallic Means, the common Greatest Prime Factor of the radical $\left(n^{2}+4\right)$ is 13. Also, for $1^{\text {st }}, 4^{\text {th }}, 6^{\text {th }}, 11^{\text {th }}$ Metallic Means, the common Greatest Prime Factor of the radical $\left(n^{2}+4\right)$ is $\mathbf{5}$. 
Hence, the different Metallic Means can be classified into various groups corresponding to the Greatest Prime Factors (GPF) of the radical $\left(\mathbf{n}^{2}+\mathbf{4}\right)$. This GPF is necessarily a Pythagorean Prime $(4 x+1)$, as shown below in Table 4.

Table 4 : Prime Families of Metallic Means

\begin{tabular}{|c|c|}
\hline $\begin{array}{c}\text { Greatest Prime Factor } \\
(\text { GPF }) \\
\text { of }\left[n^{2}+4\right]\end{array}$ & $\begin{array}{c}\mathbf{n}_{\mathbf{s}} \text { of the associated } \\
\text { Metallic Means }\left(\boldsymbol{\delta}_{\mathrm{n}}\right)\end{array}$ \\
\hline 5 & $1,4,6,11$ \\
\hline 13 & $3,10,16,29$ \\
\hline 17 & $8,9,26,43$ \\
\hline 29 & $12,25,49$ \\
\hline 37 & $18,23,59$ \\
\hline 41 & \\
\hline
\end{tabular}

Noticeably, the Hypotenuse of associated Pythagorean Triple is a factor of $\left(n^{2}+4\right)$, and the associated Pythagorean Primes, as shown in Table 4 are the Greatest Prime Factors of $\left(n^{2}+4\right)$. Hence note: the $8^{\text {th }}$ and the $9^{\text {th }}$ Metallic Means both have Pythagorean Prime 17 as the GPF of their $\left(n^{2}+4\right)$, however they have different associated Primitive Pythagorean Triples, as shown in Table 2.

Moreover, beside the Greatest Prime Factors indicated in above Table, multuple Pythagorean Primes constitute the factors of various $\left(\mathrm{n}^{2}+4\right)$ radicals.

$(59)^{2}+4=(41 \times 17 \times 5)$

$(49)^{2}+4=(37 \times 13 \times 5)$, and so on.

Noticeably, the Greatest Prime Factor for $n=43$ is 109

$(43)^{2}+4=109 \times 17$

However, why it has been includen in the Prime 17 Family in above Table, that will become obvious with following couple of illustrations. 
Remarkably, the Metallic Means belonging to same Prime Family exhibit very distinctive relations among themselves, as illustrated below.

For instance, consider the Prime Family of Metallic Means associated with the Pythagorean Prime $\mathbf{1 3}: \boldsymbol{\delta}_{3}, \boldsymbol{\delta}_{10}$ and $\boldsymbol{\delta}_{16}$

Metallic Means associated with the Pythagorean Prime $13: \boldsymbol{\delta}_{3}, \boldsymbol{\delta}_{10}$ and $\boldsymbol{\delta}_{16}$

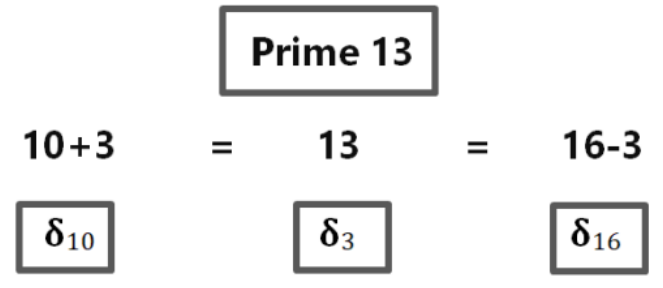

Hence, the Pythagorean Prime of this Family $13=\frac{3^{2}+4}{1}:$ Note the Digit 1 in Denominator.

Here, the $3^{\text {rd }}$ and $(3+1)^{\text {th }}$ Metallic Means give the precise value of $16^{\text {th }}$ Mean : $\frac{\boldsymbol{\delta}_{4} \times \boldsymbol{\delta}_{3}+\mathbf{1}}{\boldsymbol{\delta}_{4}-\boldsymbol{\delta}_{3}}=\boldsymbol{\delta}_{16}$

Similarly, the $3^{\text {rd }}$ and $(3-1)^{\text {th }}$ Metallic Means give the precise value of $10^{\text {th }}$ Mean : $\frac{\boldsymbol{\delta}_{3} \times \boldsymbol{\delta}_{2}+\mathbf{1}}{\boldsymbol{\delta}_{3}-\boldsymbol{\delta}_{2}}=\boldsymbol{\delta}_{10}$

Moreover, $\frac{\mathbf{1 3}-\mathbf{1}}{\mathbf{2}}=6$ :this 6 forms a new Triad with members 10 and 16 of the family: $\left[\boldsymbol{\delta}_{6}, \boldsymbol{\delta}_{10}, \boldsymbol{\delta}_{16}\right]$ :

$$
\frac{\boldsymbol{\delta}_{16} \times \boldsymbol{\delta}_{6}+\mathbf{1}}{\boldsymbol{\delta}_{16}-\boldsymbol{\delta}_{6}}=\boldsymbol{\delta}_{10} \quad \text { and } \quad \frac{\boldsymbol{\delta}_{10} \times \boldsymbol{\delta}_{6}+\mathbf{1}}{\boldsymbol{\delta}_{10}-\boldsymbol{\delta}_{6}}=\boldsymbol{\delta}_{16}
$$

Further, beyond 3, 10 and 16, more members would be added in the family of Pythagorean Prime 17, next member in the family would be 29 which equals the sum of all smaller members in the family;

$3+10+16=29$ and, the Prime $13=29-16$

And,

$2 \times 16+3+1=36$ :this 36 forms a new Triad with members 16 and 29 of the family $\left[\boldsymbol{\delta}_{16}, \boldsymbol{\delta}_{29}, \boldsymbol{\delta}_{36}\right]$ :

$$
\frac{\boldsymbol{\delta}_{36} \times \boldsymbol{\delta}_{16}+\mathbf{1}}{\boldsymbol{\delta}_{36}-\boldsymbol{\delta}_{16}}=\boldsymbol{\delta}_{29} \quad \text { and } \quad \frac{\boldsymbol{\delta}_{29} \times \boldsymbol{\delta}_{16}+\mathbf{1}}{\boldsymbol{\delta}_{29}-\boldsymbol{\delta}_{16}}=\boldsymbol{\delta}_{36}
$$


Likewise, consider another example for illustration and comparison. the Metallic Means associated with the Pythagorean Prime $17: \boldsymbol{\delta}_{8}, \boldsymbol{\delta}_{9}$ and $\boldsymbol{\delta}_{26}$

Metallic Means associated with the Pythagorean Prime $17: \boldsymbol{\delta}_{8}, \boldsymbol{\delta}_{9}$ and $\boldsymbol{\delta}_{26}$

\section{Prime 17}

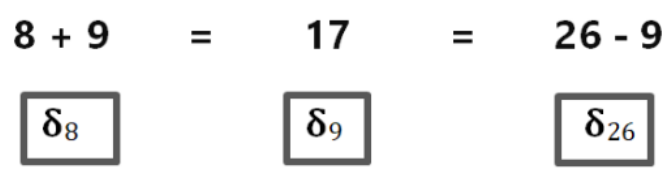

And, the Prime $17=\frac{9^{2}+4}{5}:$ Note the Digit 5 in Denominator.

Here, the $\mathbf{9}^{\text {th }}$ and $(\mathbf{9}+5)^{\text {th }}$ Metallic Means give the precise value of $26^{\text {th }}$ Mean : $\frac{\boldsymbol{\delta}_{9} \times \boldsymbol{\delta}_{14}+\mathbf{1}}{\boldsymbol{\delta}_{14}-\boldsymbol{\delta}_{9}}=\boldsymbol{\delta}_{26}$

Similarly, the $\mathbf{9}^{\text {th }}$ and $(\mathbf{9}-\mathbf{5})^{\text {th }}$ Metallic Means give the precise value of $\boldsymbol{8}^{\text {th }}$ Mean : $\frac{\boldsymbol{\delta}_{9} \times \boldsymbol{\delta}_{4}+\mathbf{1}}{\boldsymbol{\delta}_{9}-\boldsymbol{\delta}_{4}}=\boldsymbol{\delta}_{8}$

Moreover, $\frac{\mathbf{1 7}-\mathbf{5}}{\mathbf{2}}=6$ : this 6 forms a new Triad with members 8 and 26 of the family: $\left[\boldsymbol{\delta}_{6}, \boldsymbol{\delta}_{8}, \boldsymbol{\delta}_{26}\right]$

$$
\frac{\boldsymbol{\delta}_{26} \times \boldsymbol{\delta}_{6}+\mathbf{1}}{\boldsymbol{\delta}_{26}-\boldsymbol{\delta}_{6}}=\boldsymbol{\delta}_{8} \quad \text { and } \quad \frac{\boldsymbol{\delta}_{8} \times \boldsymbol{\delta}_{6}+\mathbf{1}}{\boldsymbol{\delta}_{8}-\boldsymbol{\delta}_{6}}=\boldsymbol{\delta}_{26}
$$

Further, beyond 8,9 and 26, more members would be added in the family of Pythagorean Prime 17, like next member in the family would be 43 which equals the sum of all smaller members in the family;

$8+9+26=43$ and, the Prime $17=43-26$

And,

$2 \times 26+9+5=66$ :this 66 forms a new Triad with members 26 and 43 of the family: $\left[\boldsymbol{\delta}_{26}, \boldsymbol{\delta}_{43}, \boldsymbol{\delta}_{66}\right]$

$$
\frac{\boldsymbol{\delta}_{66} \times \boldsymbol{\delta}_{26}+\mathbf{1}}{\boldsymbol{\delta}_{66}-\boldsymbol{\delta}_{26}}=\boldsymbol{\delta}_{43} \quad \text { and } \quad \frac{\boldsymbol{\delta}_{43} \times \boldsymbol{\delta}_{26}+\mathbf{1}}{\boldsymbol{\delta}_{43}-\boldsymbol{\delta}_{26}}=\boldsymbol{\delta}_{66}
$$

Such several distinctive correlations are observed among the Metallic Means belonging to the same Pythagorean Prime Families, and these correlations are bound to generate more such intriguing mathematical formulae, which may provide the precise relations between different Metallic Ratios.






\section{Special Pattern regarding Digits 3, 6 and 9 in the Realm of Metallic Means :}

The proponents of Vortex Based Mathematics will continue to make irrational claims, and the opponents will continue to debunk them on grounds of the Base-10 Number System. Let the both camps do their jobs with missionary zeal.

Author's objective is just to appreciate the beauty of numbers and the special attributes of the digits 3,6 and 9, especially their unique patterns in the realm of Metallic Means.

For instance, consider the Integer Sequences and the corresponding Lucas Sequences associated with various Metallic Means [11] [12]. Remarkably, the Digital Roots of every Fourth Terms of Fibonacci, Lucas, Pell and Pell-Lucas sequences are 3, 6 or 9. $\mathrm{F}_{4}, \mathrm{~F}_{8}, \mathrm{~F}_{12} \ldots \ldots . ., \mathrm{L}_{2}, \mathrm{~L}_{6}, \mathrm{~L}_{10} \ldots \ldots . ., \mathrm{P}_{4}, \mathrm{P}_{8}, \mathrm{P}_{12} \ldots \ldots \ldots$ and $\mathrm{PL}_{2}, \mathrm{PL}_{6}, \mathrm{PL}_{10} \ldots \ldots$. all have their Digital Roots 3,6 or 9 , and it holds true for the Integer Sequences as well as corresponding Lucas Sequences associated with any $\mathrm{n}^{\text {th }}$ Metallic Mean $\boldsymbol{\delta}_{\mathrm{n}}$; provided $\mathrm{n}$ is not multiple of 3 .

If $\mathrm{n}$ is multiple of 3 i.e. digital root of $\mathrm{n}$ itself is 3,6 or 9 , like Bronze Ratio $\boldsymbol{\delta}_{3}$ or the Aluminium Ratio $\boldsymbol{\delta}_{6}$ or the Ninth Mean $\boldsymbol{\delta}_{9}$ or the Twelfth Mean $\boldsymbol{\delta}_{12}$ and so on : here, not just every fourth, but the Alternate terms of associated Integer Sequences and corresponding Lucas Sequences have the digital roots 3, 6, or 9 .

Now, lets incorporate here the idiosyncratic formula $\frac{\boldsymbol{\delta}_{\mathrm{m}} \times \boldsymbol{\delta}_{\mathrm{n}}+\mathbf{1}}{\boldsymbol{\delta}_{\mathrm{m}}-\boldsymbol{\delta}_{\mathrm{n}}}=\boldsymbol{\delta}_{\mathrm{k}}$

For instance, consider the Table 1 provided on the Fourth and Fifth page of this paper.

It is noticeable in the Table $1:$ if $\mathrm{n}$ is not multiple of 3, the alternate values of $\mathrm{m}$ and $\mathrm{k}$ have their digital roots 3,6 , or 9.

And, if $\mathrm{n}$ is multiple of $3:$ None of the associated $\mathrm{m}_{\mathrm{s}}$ and $\mathrm{k}_{\mathrm{s}}$ have their digital roots 3 , 6, or 9 .

More remarkably, the number of Triples ( or the numbers of $m_{s}$ and $k_{s}$ ) increase noticeably for $n=6$ and 9

For Even $n_{s}$ : the number of Triples exhibit noticeable rise at $n=6,16,26 \ldots \ldots$ and so on.

For Odd $n_{s}$ : the number of Triples exhibit noticeable rise at $n=9,19,29 \ldots \ldots$. and so on.

For illustration of more such intriguing patterns embedded in the metallic Means, consider following couple of examples, based upon the formula $\frac{\boldsymbol{\delta}_{\mathrm{m}} \times \boldsymbol{\delta}_{\mathbf{n}}+\mathbf{1}}{\boldsymbol{\delta}_{\mathrm{m}}-\boldsymbol{\delta}_{\mathbf{n}}}=\boldsymbol{\delta}_{\mathrm{k}}$

Consider the Triads of Metallic Means formed with $\mathrm{n}=6$, as shown below.

\begin{tabular}{|c|c|c|c|c|c|c|c|c|}
\hline$n$ & 6 & 6 & 6 & 6 & 6 & 6 & 6 & 6 \\
\hline$m$ & 7 & 8 & 10 & 11 & 14 & 16 & 26 & 46 \\
\hline$m-n$ & 1 & 2 & 4 & 5 & 8 & 10 & 20 & 40 \\
\hline
\end{tabular}


Note the bottom row in above table which contains the values of $(\mathbf{m}-\mathbf{n})$.

The numbers in this $(m-n)$ row exhibit $1-2-4-5-8-10 \times(1-2-4)$ pattern, and remarkably the digits 3,6 and 9 are conspicuous by their absence from this row!

And what's about digit 7 ? Consider another example with $n=34$, as shown below.

\begin{tabular}{|c|l|l|l|l|l|l|l|l|l|l|l|l|l|l|l|l|}
\hline $\mathrm{n}$ & 34 & 34 & 34 & 34 & 34 & 34 & 34 & 34 & 34 & 34 & 34 & 34 & 34 & 34 & 34 & 34 \\
\hline $\mathrm{m}$ & 35 & 36 & 38 & 39 & 42 & 44 & 54 & 63 & 74 & 92 & 150 & 179 & 266 & 324 & 614 & 1194 \\
\hline $\mathrm{m}-\mathrm{n}$ & 1 & 2 & 4 & 5 & 8 & 10 & 20 & 29 & 40 & 58 & 116 & 145 & 232 & 290 & 580 & 1160 \\
\hline
\end{tabular}

As any $\mathrm{n}^{\text {th }}$ Metallic Mean $\boldsymbol{\delta}_{\mathrm{n}}$ can give the precise values of other Metallic Means $\boldsymbol{\delta}_{\mathrm{m}}$ and $\boldsymbol{\delta}_{\mathrm{k}}$ by the formula:

$\frac{\boldsymbol{\delta}_{\mathbf{m}} \times \boldsymbol{\delta}_{\mathbf{n}}+\mathbf{1}}{\boldsymbol{\delta}_{\mathbf{m}}-\boldsymbol{\delta}_{\mathbf{n}}}=\boldsymbol{\delta}_{\mathbf{k}} ;$ with $\mathbf{k}_{\max }=\mathbf{m}_{\max }=\mathrm{n}^{2}+\mathrm{n}+4 ;$

Hence, $(m-n)_{\max }=\left(\mathbf{n}^{\mathbf{2}}+\mathbf{4}\right)$ which is the all important Radical in the fractional expression of $\mathrm{n}^{\text {th }}$ Metallic Mean $\boldsymbol{\delta}_{\mathrm{n}}$

In above table with $\mathrm{n}=34,(\mathrm{~m}-\mathrm{n})_{\max }=1160$

Consider the Prime Factorization of this (m-n) $\max : 1160=1 \times 2 \times 2 \times 2 \times 5 \times 29$

Note the bottom row $(m-n)$ in above table, the numbers in the row exhibit the characteristic pattern based upon these factors $1,2,5$ and 29 . Numbers in the row are the $1-2-4-5-8-10 \times(1-2-4)$ multiples of the prime factors $1,2,5$ and 29.

Noticeably, the digit 7 is present not directly as $(m-n)$, but it's present only as the Digital Roots of certain $(m-n)$ values; for instance the red shaded number 232 in above example. Such presence of 7 as Digital Root of (m-n) values is observed with $n=8,10,11,14,16,22,26,29,34,36,39$, and so on. Remarkably, with $n=26,36$, 39, etc. the digital roots of multiple $(m-n)$ values are found to be 7 .

However, the digits 3, 6 and 9 are invariably missing from this pattern, they are neither present directly, nor as the digital roots of any $(m-n)$ or $(k-n)$ values.

For Even $\mathrm{n}_{\mathrm{s}}$ : the typical $1-2-4-5-8-10 \times(1-2-4)$ pattern is observed.

For Odd $n_{s}$ : pattern based upon product of the Prime Factors of $\left(n^{2}+4\right)$ is observed.

In either case, the digits 3, 6 and 9 are conspicuous by their absence from these $(m-n)$ or $(k-n)$ values.

Moreover, if $n$ is multiple of $3:$ the digital roots of $[(m-n)+(k-n)]$ are invariably $4-5-4-5$

And, if $n$ is not multiple of 3 : the digital roots of $[(m-n)+(k-n)]$ are invariably $\mathbf{3}-\mathbf{6}-\mathbf{9}$, as shown below. 
For example, consider $\mathrm{n}=30$ ( digital root of $\mathrm{n}$ is 3 )

\begin{tabular}{|c|c|c|c|c|c|c|c|c|}
\hline $\mathbf{n}$ & 30 & 30 & 30 & 30 & 30 & 30 & 30 & 30 \\
\hline $\mathbf{m}$ & 31 & 32 & 34 & 38 & 143 & 256 & 482 & 934 \\
\hline $\mathbf{k}$ & 934 & 482 & 256 & 143 & 38 & 34 & 32 & 31 \\
\hline $\mathbf{m}-\mathbf{n}$ & 1 & 2 & 4 & 8 & 113 & 226 & 452 & 904 \\
\hline $\mathbf{k}-\mathbf{n}$ & 904 & 452 & 226 & 113 & 8 & 4 & 2 & 1 \\
\hline Digital Root of (m-n)+(k-n) & $\mathbf{5}$ & $\mathbf{4}$ & $\mathbf{5}$ & $\mathbf{4}$ & $\mathbf{4}$ & $\mathbf{5}$ & $\mathbf{4}$ & $\mathbf{5}$ \\
\hline
\end{tabular}

Likewise, consider $\mathrm{n}=29$ (digital root of $\mathrm{n}$ is other than 3,6 or 9 )

\begin{tabular}{|c|c|c|c|c|c|c|}
\hline $\mathbf{n}$ & 29 & 29 & 29 & 29 & 29 & 29 \\
\hline $\mathbf{m}$ & 30 & 34 & 42 & 94 & 198 & 874 \\
\hline $\mathbf{k}$ & 874 & 198 & 94 & 42 & 34 & 30 \\
\hline $\mathbf{m}-\mathbf{n}$ & 1 & 5 & 13 & 65 & 169 & 845 \\
\hline $\mathbf{k}-\mathbf{n}$ & 845 & 169 & 65 & 13 & 5 & 1 \\
\hline Digital Root of (m-n)+(k-n) & $\mathbf{9}$ & $\mathbf{3}$ & $\mathbf{6}$ & $\mathbf{6}$ & $\mathbf{3}$ & $\mathbf{9}$ \\
\hline
\end{tabular}

\section{Conclusion:}

This paper apposed and synergised the newly discovered couple of aspects of the Metallic Ratios, viz. the generalised geometry of Metallic Means premised upon Right Triangles and the mathematical correlation between different Metallic Ratios. And these two novel aspects gave rise to the formations of the distinctive Triads of Metallic Means. This paper also unveiled the close associations between such Right Triangles representing Metallic Ratios and the primitive Pythagorean Triples, as well as the close correspondence between the Metallic Means and Pythagorean Primes. Moreover, this work illustrated the intriguing patterns of Digits 369 in realm of Metallic Numbers. Triangles Triads -Triples - \& 369 .....!

\section{References:}

[1] Rajput, Chetansing (2021). "A Right Angled Triangle for each Metallic Mean". Journal of Advances in Mathematics. 20: 32-33. https://en.wikipedia.org/wiki/Metallic mean\#cite note-15 
[2] Vera W. de Spinadel (1999). The Family of Metallic Means, Vismath 1(3) from Mathematical Institute of Serbian Academy of Sciences and Arts.

[3] Weisstein, Eric W. "Table of Silver means". MathWorld.

[4] "An Introduction to Continued Fractions: The Silver Means", maths.surrey.ac.uk.

[5] Rajput, Chetansing (2021). Golden Ratio. JOURNAL OF ADVANCES IN MATHEMATICS, 20, $19-42$. https://doi.org/10.24297/jam.v20i.8945

[6] Rajput, Chetansing (2021). Metallic Ratios : Beyond the Golden Ratio; The Mathematical Relationships between different Metallic Means. JOURNAL OF ADVANCES IN MATHEMATICS, 20, $158-166$. https://doi.org/10.24297/jam.v20i.9023

[7] Polygons, Diagonals, and the Bronze Mean by Antonia R. Buitrago

[8] M, Teller. "Polygons \& Metallic Means". tellerm.com. Retrieved 2020-02-05.

[9] Rajput, Chetansing (2021). Metallic Means and Right Triangles: The Geometric Substantiation of all Metallic Ratios JOURNAL OF ADVANCES IN MATHEMATICS, 20, 167-173. https://doi.org/10.24297/jam.v20i.9029

[10] Rajput, Chetansing (2021). Golden Ratio and other Metallic Means: The Geometric Substantiation of all Metallic Ratios with Right Triangles. JOURNAL OF ADVANCES IN MATHEMATICS, 20, 174-187. https://doi.org/10.24297/jam.v20i.9034

[11] Sloane, N. J. A. Sequences A000045/M0692, A000204/M2341 in "The On-Line Encyclopedia of Integer Sequences." https://oeis.org/A000045 and https://oeis.org/A000204

[12] Sloane, N. J. A. Sequences A000129/M1413, A002203/M0360, in "The On-Line Encyclopedia of Integer Sequences." http://oeis.org/A000129 and http://oeis.org/A002203

\section{About The Author :}

Dr. Chetansing K. Rajput is an M.B.B.S. Doctor from Nair Hospital (Mumbai University, India) and currently working as Asst. Commissioner (Dept. of GST, Govt of Maharashtra). Being a doctor-turned-bureaucrat, author has not received any formal education in Mathematics, does not have even any of the basic degrees in Maths. All the findings communicated in author's various publications are the results of his home based, self sponsored research pursuits, and his sincere efforts in the name of Science and Mathematics, as an amateur inventor.

Author is grateful to Wikipedia for citing his work on generalised geometric construction of all Metallic Means, in the Wiki page "Metallic Mean". That's the greatness and magnificence of Wikipedia, the free encyclopedia, which cares most about the real substance in any manuscript, rather than the so called academic degree of author. Citation by Wikipedia has really inspired and motivated the author to further discover the various aspects of Metallic Means. Thanks Wikipedia!

\section{Select Lecture Videos of Author: \\ 1) https://youtu.be/raosniXwRhw \\ 2) https://youtu.be/Qh2B1tMl8Bk \\ 3) https://youtu.be/vBfVDaFnA2k}

\section{Contacts:}

Website: https://goldenratiorajput.com/ Email: chetansingkrajput@gmail.com Email: chetansingkrajput1129@gmail.com Contacts: (+91) 7057521129 